\title{
Le vote éthique, une réponse efficace aux conflits ethniques?
}

\author{
Le cas de l'Éthiopie
}

\author{
Marie-Anne Valfort*
}

\begin{abstract}
Dans cet article, nous souhaitons comprendre si, dans une démocratie multiethnique potentiellement déchirée par la guerre civile, des préoccupations éthiques cruciales comme l'aversion à l'inégalité inter-ethnique permettent de réduire de façon significative la tentation des citoyens de voter pour leur parti ethnique (défendant l'intérêt de leur groupe ethnique au détriment des autres) plutôt que pour un parti national (promouvant un traitement équitable des différents groupes ethniques). Nous analysons pour cela des données que nous avons collectées en mai 2004 auprès de 331 étudiants de l'Université d'Addis Abeba. Nos résultats invitent à la fois au pessimisme et à l'optimisme.
\end{abstract}

\section{ETHICAL VOTING, A POWERFUL DEVICE AGAINST ETHNIC CONFLICTS? EVIDENCE FROM ETHIOPIA}

In this article, we aim at understanding whether, in multi-ethnic democracies potentially torn apart by ethnic conflicts, crucial ethical concerns like aversion towards inter-ethnic inequality may significantly lower citizens' temptation to vote for their ethnic party (promoting the interest of their ethnic group at the cost of others) rather than for a nationwide party (promoting a fair treatment of various ethnic groups). For that purpose, we analyse data that we collected in May 2004 among 331 students from Addis Ababa University. Our results yield reasons both for pessimism and optimism.

Classification JEL : D02, D63, D64, D72, H77, N47

L'effet des guerres civiles sur la croissance des pays en conflit et de leurs voisins a fait l'objet de nombreuses études qui, toutes, ont confirmé son caractère dévastateur (Alesina et al. [1996], Murdoch et Sandler [2002] et [2004]). Réduire les risques de conflits (en réduisant les risques d'oppression d'un groupe

* Laboratoire d'économétrie de l'École polytechnique et Laboratoire de macroéconomie du CREST. Courriel : marie-anne.valfort@shs.polytechnique.fr

Je remercie très chaleureusement Guy Laroque, ainsi que Maurice Baslé, Pierre Cahuc, JeanMichel Grandmont, Jean-François Laslier et Étienne Lehmann pour leurs précieux conseils. Je remercie également vivement Michael Visser, ainsi que Jean-Claude Berthélemy et Gérard Prunier pour leur lecture critique d'une version très préliminaire de cet article. Je remercie enfin la Chaire de Développement durable de l'École polytechnique pour le financement de l'enquête menée en Éthiopie dont nous analysons ici les données. 
par un autre) dans des pays déjà ravagés par la pauvreté est ainsi devenu l'une des principales priorités pour les organisations internationales à la poursuite des Objectifs du millénaire pour le développement (Humphreys et Varshney [2004]), d'autant que ces pays sont souvent ceux qui présentent par ailleurs les caractéristiques sociologiques (la pluralité ethnique notamment) les plus propices aux explosions de violence (Carment [1993]).

Jusqu'à présent, les efforts pour réduire les risques de guerres civiles se sont concentrés sur le modelage institutionnel (Lake et Rothchild [1996], Bardhan [1997]). Ce modelage a d'abord consisté, au début des années 1990, en l'introduction d'institutions démocratiques dans la plupart des pays en développement en vue notamment de les protéger contre les pouvoirs despotiques, caractéristiques de la guerre froide et à l'origine de violences incessantes contre les majorités opprimées. L'adoption d'institutions démocratiques fut prolongée par des mesures destinées à empêcher le risque d'une dictature de la majorité, via par exemple le choix de règles de partage du pouvoir assurant une représentation équilibrée des différentes tendances traversant le pays lorsque celles-ci sont très polarisées, ou encore le choix de règles électorales favorisant l'émergence de partis multiethniques. Cependant, comme le souligne Przeworski ([1991], p. 79), c'est aux citoyens in fine que revient le dernier mot dans une démocratie si bien que ce modelage institutionnel ne peut être un rempart suffisant contre les risques de conflit dès lors qu'une majorité décide de l'outrepasser : "If sovereignty resides with the people, the people can decide to undermine all the guarantees reached by politicians around a negotiation table. Even the best institutionalized guarantees give at best a high degree of assurance, never certainty. »)

Ce constat constitue une invitation claire à compléter le modelage de l' " offre » politique par des mesures cherchant à influencer la "demande » politique, et notamment le sens civique du citoyen censé l'aider à renoncer à soutenir des politiques satisfaisant son intérêt à court terme précisément parce qu'elles sont iniques à l'égard des autres groupes composant la société. Mais pour espérer influencer ce sens civique, encore faut-il qu'il joue effectivement un rôle dans les préférences politiques qui seront exprimées par le citoyen à travers son vote, c'est-à-dire que le vote du citoyen soit, au moins partiellement, «éthique » dans le sens donné par Goodin et Robert [1975] où il est mû par la volonté de garantir la justice et donc la concorde civile à long terme. Plus précisément, cet article tente de répondre à la question de recherche suivante: "Dans une démocratie multiethnique potentiellement déchirée par la guerre civile, des préoccupations éthiques cruciales comme l'aversion à l'inégalité inter-ethnique permettent-elles de réduire de façon significative la tentation des citoyens de voter pour leur parti ethnique (défendant l'intérêt de leur groupe ethnique au détriment des autres) plutôt que pour un parti national (promouvant un traitement équitable des différents groupes ethniques)?»

Notre article s'organise en trois temps. Nous présentons d'abord les données que nous avons collectées en Éthiopie en mai 2004. Nous spécifions ensuite notre modèle économétrique et définissons les variables dépendantes et explicatives clés. Nous présentons enfin les résultats de notre régression et concluons notre article en insistant sur leurs implications en terme de développement. 


\section{LES DONNÉES}

\section{Pourquoi l'Éthiopie?}

De nombreux pays auraient pu constituer des terrains d'étude pertinents pour nous aider à répondre à notre question de recherche. Cependant, l'Éthiopie est sans doute l'un des pays où la tension entre « vote ethnique » et « vote national » est actuellement la plus forte. En effet, l'adoption en 1994 d'une forme nouvelle d'organisation de l'État en Afrique sub-saharienne, le fédéralisme ethnique ${ }^{1}$, a contribué à « ethniciser » la vie politique éthiopienne en aboutissant à la division des partis politiques le long de clivages ethniques, mais aussi en radicalisant le discours pro-ethnique de chacun.

Trois raisons inhérentes au fédéralisme ethnique ou à son contexte expliquent cette tendance. Tout d'abord, le fédéralisme ethnique crée les conditions de l'« ethnicisation des esprits » (Vaughan [2003] et [2005]) en figeant l'ethnicité à l'intérieur de frontières institutionnalisées alors même que l'appartenance ethnique est par définition un phénomène anthropologique en perpétuelle évolution. Par ailleurs, l'adoption du fédéralisme ethnique a souvent été dénoncée comme " prématurée » (Abbink [1997]) car elle s'est déroulée sans que soit organisée au préalable une réflexion nationale sur ce qui continuait à unir la population éthiopienne après des décennies d'oppression d'une majorité par une minorité (l'empire Amhara de Ménelik et d'Hailé Sélassié (1889-1974), puis le despotisme centralisateur de Mengistu (1974-1991)). Cette absence de débat de fond explique que l'instauration du fédéralisme ethnique ait favorisé la primauté de la « pensée ethnique » sur la «pensée nationale ». Enfin, en raison d'inégalités régionales très fortes, le fédéralisme ethnique n'a pu être accompagné d'une décentralisation effective de la gestion des ressources nationales. D'après la Banque mondiale [2001], le gouvernement fédéral centralise ainsi $80 \%$ des revenus fiscaux $(60 \%$ des revenus totaux) ainsi que la totalité de l'aide et des prêts en provenance des institutions internationales (20 à $30 \%$ des revenus totaux). Dans un contexte où l'EPRDF, la coalition au pouvoir en place depuis 1991, est dominée par le parti tigréen TPLF, cette centralisation des ressources semble avoir facilité leur allocation dans un sens favorable à la région du Tigré et à ses élites (favoritisme ethnique dénoncé notamment par Aalen [2001], Gudina [2003] et un ancien membre du TPLF interviewé par Mesfin [2006]) ainsi qu'aux « petites » régions ${ }^{2}$ qui ne sont pas gouvernées par des partis membres de l'EPRDF (clientélisme politique dénoncé par Gudina [2003]). L'analyse plus précise de la formule utilisée pour répartir les ressources entre les différentes régions fédérales (Banque mondiale [2001]) semble confirmer ces dérives ethnique et clientéliste puisqu'elle privilégie clairement les « petites » régions dont le Tigré ( $7 \%$ de la population éthiopienne $\left.{ }^{3}\right)$ fait

1. Le fédéralisme ethnique a abouti à la création de neuf régions fédérales délimitées sur des bases essentiellement ethniques (il s'agit du Tigré, de l'Afar, de l'Amhara, de l'Oromiya, de l'État Somali, du Benishangul-Gumuz, de la région des PNNS (Peuples, Nations, et Nationalités du Sud), du Gambella et de 1'Harari), et deux zones administratives : Dire Dawa et Addis Abeba.

2. Il s'agit de la région Somali, du Benishangul-Gumuz, du Gambella, de l'Afar, de l'Harari, et de Dire Dawa qui regroupent au total moins de $10 \%$ de la population éthiopienne.

3. Le maintien au pouvoir de l'EPRDF paraît dès lors difficilement compréhensible, à moins de rappeler les moyens de pression dont disposent les autorités éthiopiennes sur les populations rurales ( $85 \%$ de la population totale), parmi lesquels on compte la fourniture de nourriture (en cas de sécheresse), d'engrais, de semences, etc., sous condition de soutien politique (Pausewang et al. [2003]). 
partie. Ainsi, alors que la région du Tigré affiche des niveaux de pauvreté quasi équivalents à ceux de la région Amhara et de la région des PNNS $(57,9 \%, 56,7 \%$ et $56,5 \%$ de leur population respectivement vit en dessous du seuil de pauvreté d'après Bigsten et al. [2005]), le Tigré reçoit, sur la période 1993-2001, une allocation par tête entre 1,5 fois et 2 fois supérieure à l'allocation par tête à destination de l'Amhara et de la région des PNNS.

Le ressentiment éprouvé de la part des groupes ethniques visiblement discriminés s'est traduit par l'émergence de partis d'opposition ethniques, dont notamment l'AAPO (le parti pro-Amhara), le SEPDC (le parti pro-PNNS), l'ONC et l'OLF (les partis pro-Oromo). Bien que chacun de ces partis défendent des politiques favorisant prioritairement leur groupe ethnique, ce sont sans doute les partis oromos qui affichent le discours pro-ethnique le plus radical. En effet, les Oromos constituent la plus grande ethnie du pays ( $35 \%$ de la population) ce qui, à condition d'un fonctionnement irréprochable des institutions démocratiques et d'un vote massivement ethnique de la part des populations oromos, leur garantirait le plus grand nombre de sièges à la Chambre des représentants du peuple ${ }^{1}$. Par ailleurs, l'Oromiya est considérée comme le grenier de l'Éthiopie, ce qui rend les menaces de sécession formulées notamment par l'OLF particulièrement crédibles.

Pourtant, en marge de ces partis ethniques, une opposition composée de partis " nationaux » défendant un traitement égalitaire des différents groupes ethniques a émergé à la fin des années 1990, et bénéficie d'un soutien très important au sein de la population d'Addis Abeba. Deux raisons principales expliquent ce soutien. D'abord, Addis Abeba affiche une très forte diversité ethnique ce qui, sous l'hypothèse que les partis pro-ethniques favorisent prioritairement leur région ethnique en cas d'accès au pouvoir, rend le vote ethnique peu incitatif pour les habitants d'Addis Abeba. Par ailleurs, Addis Abeba est la seule zone qui ne reçoit aucune subvention de la part du gouvernement fédéral. En d'autres termes, Addis Abeba est un contributeur net à toute politique ethnique ou clientéliste potentielle, ce qui rend le vote ethnique non seulement peu incitatif, mais même coûteux.

Au terme de ce survol du paysage politique éthiopien, il semble que l'étude de l'arbitrage entre vote ethnique et vote national soit particulièrement adaptée à cette démocratie multiethnique où coexistent effectivement partis ethniques et partis nationaux.

\section{Les caractéristiques socio-démographiques de l'échantillon}

Nos données sont tirées d'un questionnaire que nous avons mené auprès de 331 étudiants de l'Université d'Addis Abeba en mai 2004. Ce questionnaire (voir Valfort [2005]) consistait en une centaine de questions destinées à connaître l'opinion des étudiants sur la démocratie, le vote, les partis politiques, l'identité

1. L'Éthiopie est un régime parlementaire bicaméral dont la chambre basse (la Chambre des représentants du peuple rassemblant 549 députés) est élue au suffrage universel. Chaque région fédérale et zone administrative y bénéficie d'un nombre fixe de sièges (croissant avec la taille de la région au-delà d'un certain seuil) qui sont attribués par scrutin majoritaire (le parti récoltant le plus grand nombre de voix dans une circonscription se voit attribuer la totalité des sièges de cette circonscription). 
nationale, etc., à la fois en tant que concepts mais aussi réalités de la vie politique éthiopienne. Les étudiants furent répartis dans six classes de 50 à 60 et répondirent au questionnaire simultanément. La durée de réponse à un questionnaire fut de 90 minutes en moyenne. Chaque participant reçut une rémunération de 30 birrs ( 10 birrs $\approx 1$ euro).

Il convient ici de souligner que la population étudiante de l'Université d'Addis Abeba constitue un échantillon très peu représentatif de la population éthiopienne (composée à $85 \%$ de ménages ruraux vivant de la culture de la terre). Cependant, elle a le mérite de présenter une diversité ethnico-régionale qu'il n'aurait pas été possible de retrouver sur un échantillon urbain ou rural puisque l'Université d'Addis Abeba accueille des étudiants issus de toute l'Éthiopie. Ainsi, seulement $21 \%$ des étudiants interviewés sont originaires d'Addis Abeba.

Plus précisément, l'échantillon regroupe des étudiants de premier et de deuxième et troisième cycles inscrits dans les cours du jour mais aussi dans les cours du soir. Parmi les 329 étudiants qui répondirent à la question « faculté », $40 \%$ sont issus de la faculté de gestion, $20 \%$ de la faculté des sciences, $13 \%$ de la faculté d'économie (les $27 \%$ restants sont inscrits en droit, en langues,... etc.). L'échantillon contient seulement $11 \%$ de femmes (soit 37 étudiantes). Le tableau 1 présente la répartition de l'échantillon en fonction de l'origine ethnique, du revenu, et de l'âge. 326 étudiants sur les 331 interviewés appartiennent à l'un des quatre principaux groupes ethniques. Les ménages « riches » (plus de 600 birrs par mois) sont légèrement surreprésentés comparé aux moyennes observées sur les populations urbaines éthiopiennes. Cependant, $25 \%$ de l'échantillon est composé d'enfants d'agriculteurs, l'accès des populations rurales aux études universitaires étant l'une des priorités du gouvernement en matière éducative.

Tableau 1. Description de l'échantillon

\begin{tabular}{|c|c|c|c|c|c|c|c|}
\hline \multicolumn{4}{|c|}{ Origine ethnique $^{a}$} & \multicolumn{4}{|c|}{$\begin{array}{l}\text { Revenu mensuel du ménage } \\
\text { (en birrs) }\end{array}$} \\
\hline & $\begin{array}{l}\text { Étudiants } \\
\%\end{array}$ & $\begin{array}{l}\text { Éthiopie } \\
\% \%\end{array}$ & & $\begin{array}{c}\text { Étudiants } \\
\%\end{array}$ & $\begin{array}{c}\text { Pop. } \\
\text { urbaine }^{c} \\
\%\end{array}$ & & $\underset{\%}{\text { Âged }^{\mathrm{d}}}$ \\
\hline Amharas & 40 & 25 & $<300$ & 40 & 45 & $18-20$ & 14 \\
\hline Oromos & 21 & 35 & $300-600$ & 22 & 25 & $21-24$ & 64 \\
\hline PNNS & 12 & 19 & $>600$ & 38 & 30 & $25-29$ & 16 \\
\hline Tigréens & 27 & $6-7$ & Total & 100 & 100 & $>30$ & 6 \\
\hline Total & 100 & $85-86$ & Tutal & 100 & 100 & Total & 100 \\
\hline
\end{tabular}

a Nous nous concentrons sur les 326 étudiants appartenant à l'un des quatre groupes principaux groupes ethniques.

b 329 étudiants ont répondu à la question « revenu » (il s'agit de celui des parents).

${ }^{\mathrm{c}}$ Données tirées de Bigsten et al. [2005].

d 323 étudiants ont répondu à la question « âge »; $\min : 18$ (âge du droit de vote) ; $\max : 42 ;$ moy : 23.3 .

\section{SPÉCIFICATION DU MODÈLE}

Nous souhaitons estimer les coefficients $\alpha_{i}(i \in[0,14])$ du modèle logit suivant : 


\begin{tabular}{cc}
\multicolumn{2}{c}{$\operatorname{Prob}($ ETHNIC_VOTE=1 $)=$} \\
\\
$1-\left\{1+\exp \left[\alpha_{0}+\right.\right.$ \\
$\alpha_{1}$ ADDIS+ & \\
$\alpha_{2}$ INEQUALITY_AVERSION+ & $\alpha_{8}$ POL_EGO + \\
$\alpha_{3}$ ETHNIC_TRUST+ & $\alpha_{9}$ TIGREAN+ \\
$\alpha_{4}$ INTERETHNIC_MARRIAGE+ & $\alpha_{10}$ OROMO + \\
$\alpha_{5}$ ETHNIC_BIAS+ & $\alpha_{11}$ AMHARA + \\
$\alpha_{6}$ NO_CREDIBLE_OPP+ & $\alpha_{12}$ INC_HH + \\
$\alpha_{7}$ ECO_DEMO_IMPROV+ & $\alpha_{13}$ AGE + \\
& $\alpha_{14}$ FEMALE $\left.]\right\}^{-1}$
\end{tabular}

La variable dépendante ETHNIC_VOTE est construite à partir de la question QC $34^{1}$ du questionnaire. Elle est égale à 1 si l'interviewé soutient l'un des partis ethniques (TPLF/EPRDF, AAPO, SEPDC, ONC et OLF) et à 0 si l'interviewé soutient l'un des partis nationaux ou s'il ne soutient aucun parti. $38 \%$ des interviewés affirment soutenir un parti ethnique (20\% des Amharas, $38 \%$ des Oromos, $18 \%$ des PNNS et $67 \%$ des Tigréens). Nous présentons dans la suite les autres variables explicatives du modèle, et notamment la variable centrale capturant l'aversion à l'inégalité inter-ethnique des individus dont nous attendons qu'elle réduise la probabilité du vote ethnique.

\section{L'aversion à l'inégalité inter-ethnique}

Nous créons deux variables d'aversion à l'inégalité inter-ethnique ( $a v_{-} I$ et $a v \_I I$ dans la suite) en vue de tester la robustesse de notre spécification. Chacune de ces variables résulte du croisement entre une variable appelée mobilisation traduisant la volonté de mobilisation de l'individu contre les éventuelles inégalités de traitement organisées par le gouvernement, et une variable appelée no_proethnic_temptation traduisant sa résistance à la tentation pro-ethnique. La prise en compte de cette dernière variable en plus de la variable mobilisation semble indispensable pour garantir que le rejet de l'éventuelle politique protigréenne du gouvernement ne coïncide pas chez l'individu avec la volonté de la remplacer par une politique favorable à son propre groupe ethnique.

La variable mobilisation est la même pour les deux variables $a v_{-} I$ et $a v_{-} I I$. Elle est construite à partir de la question QB17g $\mathrm{g}^{2}$. La variable mobilisation est égale à 1 si l'individu a répondu « Support an opposition party », « Boycott elections », ou « Join a protest », et à 0 s'il a répondu « Support the government » ou « Nothing ».

La variable no_proethnic_temptation est spécifique à chacune des variables $a v \_I$ et $a v \_I I$. La variable no_proethnic_temptation_I est construite à partir de la question QC293. Cette variable est égale à 1 si l'individu n'a pas entouré l'option «People belonging to the same ethnic group as you » et à 0 sinon. La variable

1. Which party do you support?

2. What would you be willing to do if the government distributed the wealth of the country only to those who strongly support him or who belong to the same ethnic group or economic class, to the detriment of other Ethiopian people?

3. With which of the following groups do you consider to share the highest number of interests to be politically defended? You can circle more than 1 answer [10 options proposées]. 
no_proethnic_temptation_II est construite à partir de la question QC31 ${ }^{1}$. Cette variable est égale à 1 si l'individu a répondu « More prefer candidate $\mathrm{B}$ to candidate A », « Much more prefer candidate B to candidate A » ou « Only prefer candidate $\mathrm{B} »$, et à 0 sinon.

\section{Les autres variables explicatives}

Le tableau 2 présente une rapide description des autres variables explicatives ainsi que leur fréquence (en \%) dans l'échantillon entier, et dans chaque groupe ethnique.

Tableau 2. Description des variables explicatives

\begin{tabular}{|c|c|c|}
\hline ADDIS & $=1$ si originaire d'Addis Abeba & $21^{\mathrm{a}}(23 ; 22 ; 31 ; 12.5)^{\mathrm{b}}$ \\
\hline INEQUALITY_AVERSION_I & cf: description de $a v_{-} I$ & $72(76 ; 60 ; 74 ; 74)$ \\
\hline INEQUALITY_AVERSION_II & cf: description de $a v \_I I$ & $73(75 ; 73 ; 65 ; 73)$ \\
\hline ETHNIC_TRUST & $\begin{array}{l}\text { comparaison du niveau de } \\
\text { confiance pour son groupe } \\
\text { ethnique par rapport aux autres } \\
\text { groupes ethniques }(=1 \text { si « }<» ;=2 \\
\text { si «=»;=3 si «>») }\end{array}$ & $\begin{array}{l}=1: 9(8 ; 8 ; 12 ; 9) \\
=2: 54(53 ; 46 ; 61 ; 59) \\
=3: 37(39 ; 46 ; 27 ; 31)\end{array}$ \\
\hline INTERETHNIC_MARRIAGE & $\begin{array}{l}=1 \text { si parents issus de groupes } \\
\text { ethniques différents }\end{array}$ & $30(21 ; 41 ; 36 ; 4.5)$ \\
\hline ETHNIC_BIAS & $\begin{array}{l}=1 \text { si considère son parti ethnique } \\
\text { comme « national » }\end{array}$ & $27(14 ; 10 ; 3 ; 64)$ \\
\hline NO_CREDIBLE_OPP & $\begin{array}{l}=1 \text { si pense qu'aucun autre } \\
\text { parti n'aurait pu faire mieux } \\
\text { que l'EPRDF (en terme de } \\
\text { développement économique) }\end{array}$ & $37(28 ; 29 ; 31 ; 42)$ \\
\hline ECO_DEMO_IMPROV & $\begin{array}{l}=1 \text { si pense que la situation } \\
\text { économique ainsi que la } \\
\text { démocratie se sont améliorées } \\
\text { depuis Mengistu (1974-1991) }\end{array}$ & $47(34 ; 20 ; 51 ; 83)$ \\
\hline POL_EGO & $\begin{array}{l}=1 \text { si pense que les hommes } \\
\text { politiques ne songent qu'à eux }\end{array}$ & $38(46 ; 42 ; 36 ; 23)$ \\
\hline INC_HH & $\begin{array}{l}\text { revenu des parents : =1 si } \\
\ll<300 » ;=2 \mathrm{si} \ll 300-600 » ;=3 \\
\text { si } \ll>600 »\end{array}$ & $40(35 ; 43 ; 28 ; 52)^{\mathrm{c}}$ \\
\hline AGE & codé de 1 ( $\leqslant 20$ ans $)$ à 5 (>30 ans) & $\begin{array}{l}23.3(23.9 ; 23.3 ; 22.3 ; \\
22.9)^{\mathrm{d}}\end{array}$ \\
\hline FEMALE & $=1$ si femme & $11(11 ; 12 ; 21 ; 8)$ \\
\hline \multicolumn{3}{|c|}{$\begin{array}{l}\text { a Pourcentage sur l'ensemble de l'échantillon. } \\
\text { b Pourcentage parmi les Amharas, les Oromos, les PNNS, et les Tigréens respectivement. } \\
\text { c Pourcentage des individus dont le revenu mensuel des parents est inférieur à } 300 \text { birrs. } \\
\text { d Âge moyen. }\end{array}$} \\
\hline
\end{tabular}

1. Please indicate in the following chart which of these 2 candidates (A or B) you prefer.

Candidate A: He belongs to the same ethnic group as you and promises to increase the wellbeing of people from your ethnic group first and foremost, even if this means decreasing the wellbeing of people from other ethnic groups. Candidate B: He belongs to the same ethnic group as you but promises to increase the well-being of all Ethiopian people equally, without particularly favouring your ethnic group. 
À préférences éthiques données, nous savons qu'un individu originaire d'Addis Abeba n'est jamais incité à voter pour son parti ethnique, alors qu'un individu originaire d'une région ethnique est incité à voter pour son parti ethnique. La variable ADDIS sera donc utilisée dans la suite pour capturer l'effet de l'intérêt égoïste des individus dans leur décision de voter pour leur parti ethnique plutôt que pour un parti national.

\section{LES RÉSULTATS}

Le tableau 3 présente l'estimation de notre modèle logit.

Tableau 3. Estimation du modèle logit

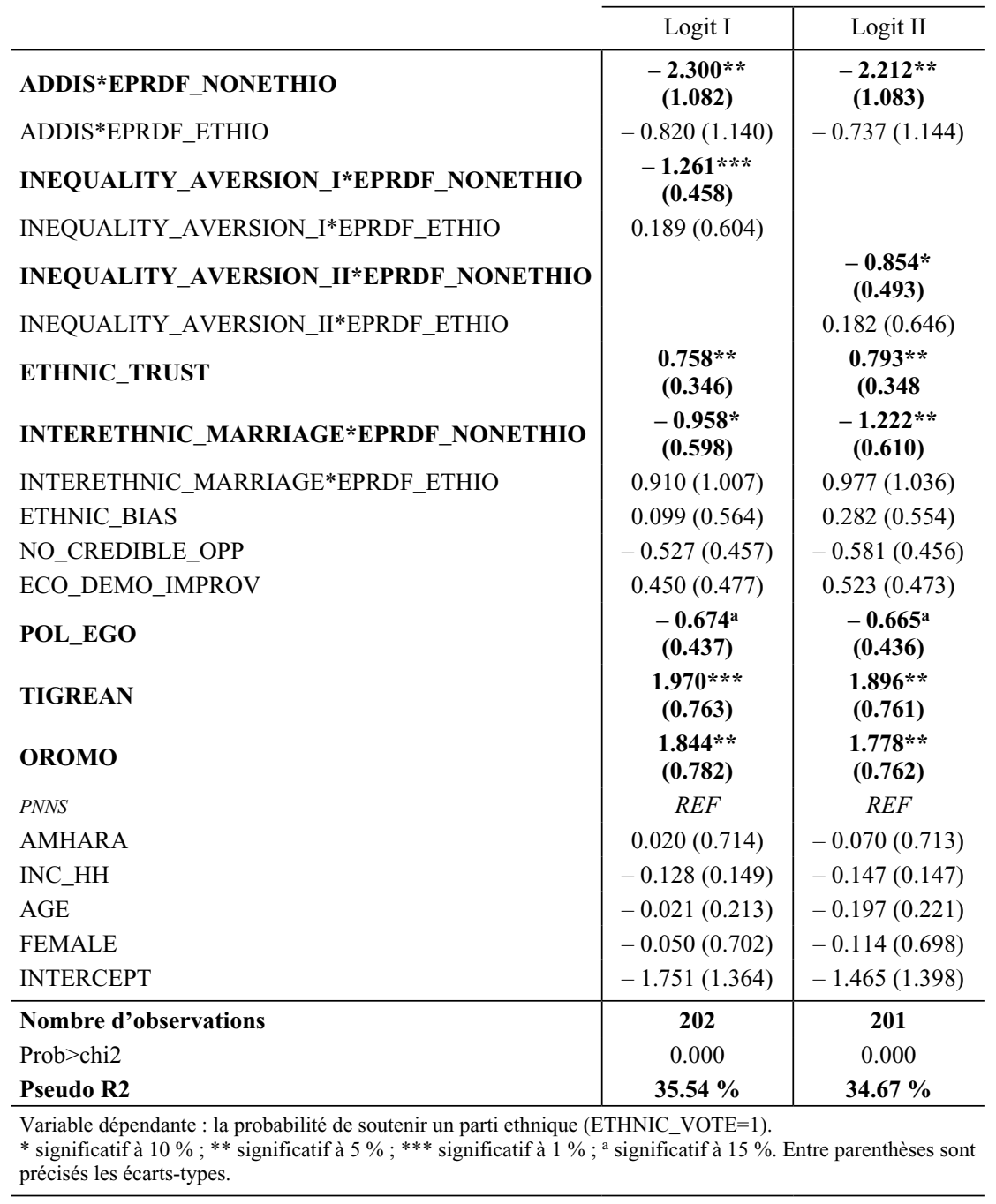


Les statistiques relatives à la variable ETHNIC_BIAS (voir tableau 2) montrent qu'une très faible minorité parmi les Amharas, les Oromos, et les PNNS considèrent leur parti ethnique comme national. En revanche, cette proportion est très forte parmi les Tigréens (64\% considèrent l'EPRDF/TPLF comme « national »). Plus généralement, l'EPRDF/TPLF, en raison de son statut de coalition, est le parti dont le caractère ethnique fait le moins consensus, bien qu'une très forte majorité de l'échantillon le considère comme non national ( $72 \%$ sur l'ensemble de l'échantillon - $82 \%$ des Amharas, $93 \%$ des Oromos, $89 \%$ des PNNS, mais $36 \%$ des Tigréens). Nous créons donc deux variables dichotomiques complémentaires (EPRDF_ETHIO et EPRDF_NONETHIO) selon que l'interviewé considère l'EPRDF/TPLF comme national ou non, et les croisons avec les variables explicatives cruciales de notre modèle. Nous nous concentrons dans l'analyse des résultats sur la valeur et la significativité des coefficients des variables croisées avec la variable EPRDF_NONETHIO puisque c'est parmi la population qui considère l'EPRDF/TPLF, l'AAPO, le SEPDC, l'ONC, et l'OLF comme non nationaux que fait sens notre spécification (i.e : l'étude de l'arbitrage entre vote ethnique et vote national).

\section{CONCLUSION}

Nos résultats invitent à la fois au pessimisme et à l'optimisme. Notons que, la population éthiopienne étant composée d'individus en moyenne beaucoup moins éduqués et beaucoup plus pauvres que les étudiants constituant notre échantillon, il semble raisonnable de penser que la teneur pessimiste de nos conclusions serait renforcée si elles étaient tirées de l'étude d'un échantillon plus représentatif de la population éthiopienne.

Nos résultats invitent au pessimisme puisque, en moyenne, l'influence marginale des considérations éthiques (coefficients des variables INEQUALITY AVERSION) apparaît environ deux fois plus faible que l'influence marginale de l'intérêt égoïste des individus (coefficient de la variable ADDIS), sans compter que l'impact des motivations éthiques est sans doute surestimé dans notre régression. En effet, la relation entre les variables INEQUALITY_AVERSION et ETHNIC_VOTE ne peut être considérée comme uniquement causale. La prédominance des motivations égoïstes apparaît d'autant plus forte de la part des groupes ethniques (les Tigréens et les Oromos) dont la compétition est la plus âpre et donc la plus déstabilisante pour l'Éthiopie dans son ensemble. En d'autres termes, le sens civique des citoyens ne peut à lui seul les faire renoncer au vote ethnique, ce qui implique, comme l'affirme Bardhan [1997, p 1392], de le compléter par le modelage du cadre institutionnel si l'on veut réduire les risques de conflits ethniques : « Working toward an incentive structure that induces otherwise disaffected people to patch up and cooperate is more useful than mere sermons about solidarity and fraternity. "

Malgré cela, nos résultats offrent des raisons d'optimisme puisque les motivations éthiques paraissent réduire la tentation du vote ethnique de façon significative. Autrement dit, renforcer le sens civique des citoyens, en favorisant notamment le brassage ethnique, paraît constituer une stratégie prometteuse sur le long terme. La régression des variables INEQUALITY_AVERSION sur les 
variables explicatives exogènes montre en effet qu'elles dépendent positivement et significativement (à $10 \%$ ) du fait d'être issu d'un mariage interethnique.

Cependant, il est crucial que l'éveil des consciences citoyennes soit accompagné de politiques de réduction de la pauvreté pour être efficace. Les variables INEQUALITY_AVERSION dépendent ainsi positivement et significativement (à $10 \%$ ) du revenu des parents. Et effectivement, il paraît vain d'attendre un quelconque vote éthique de la part de citoyens au ventre vide.

\section{RÉFÉRENCES BIBLIOGRAPHIQUES}

AALEN L. [2001], « Ethnic federalism in a dominant party state: the Ethiopian experience (1991-2000) », Development Studies and Human Rights Report 2, Chr. Michelsen Institute.

ABBINK J. [1997], « Ethnicity and constitutionalism in contemporary Ethiopia », Journal of African Law, 41 (2), p. 159-174.

Alesina A., OetZler S., Roubini N. et Swagel P. [1996], «Political instability and economic growth », Journal of Economic Growth, 1 (2), p. 189-211.

BANQUE MONDIALE [2001], Ethiopia : focussing public expenditures on poverty reduction, Report No. 232351-ET, Volume I et II.

BARDHAN P. [1997], « Method in the madness? A political-economy analysis of the ethnic conflicts in less developed countries », World Development, 25 (9), p. 1381-1398.

Bigsten A., Kebede B. et Shimeles A. [2005], Poverty, income distribution and labour markets in Ethiopia, Nordiska Afrikainstitutet.

CARMENT D. [1993], « The international dimension of ethnic conflict: concepts, indicators, and theory », Journal of Peace Research, 30 (2), p. 137-150.

Goodin R. et RoberTs K. [1975], «The ethical voter », American Political Science Review, 69 (3), p. 926-928.

Gudina M. [2003], Ethiopia: Competing ethnic nationalisms and the quest for democracy, 1960-2000, Chamber Printing House, Ethiopia.

HuMPHREYS M. et VARSHNEY A. [2004], « Violent conflict and the Millennium Development Goals: Diagnosis and Recommandations », Draft prepared for the Meeting of the MDGS Poverty Task Force, Bangkok.

LAKE R. et ROTHCHILD D. [1996], "Containing fear: the origins and management of ethnic conflict », International Security, 21 (2), p. 41-75.

MESFIN B. [2006], « Political parties in post 1991 Ethiopia », Working Paper, Université d'Addis Abeba et Université de Grenoble.

MuRdoch J. et SANDLER T. [2002], « Economic growth, civil wars, and spatial spillovers », Journal of Conflict Resolution, 46 (1), p. 91-110.

MuRdoch J. et SANDLER T. [2004], " Civil wars and economic growth: spatial dispersion », American Journal of Political Science, 48 (1), p. 138-151.

Pausewang S., Tronvoll K., Aalen L. [2003], Ethiopia since the Derg. A decade of democratic pretension and performance, Zed Books.

PRZEWORSKI A. [1991], Democracy and the market, Cambridge University Press, Cambridge.

VALFORT M. A. [2005], « Democracy and voting survey: first results from Ethiopia », Cahier du Laboratoire d'économétrie de l'École polytechnique, 5, p. 1-69.

Vaughan S. [2003], Ethnicity and power in Ethiopia, PhD thesis, The University of Edinburgh.

VAUGHAN S. [2005], «Identité ethnique et fédéralisme ethnique en Éthiopie du Sud », Politique africaine, 99 (4), p. 22-42. 